\title{
Analysis of metabolome data by a maximum likelihood approach
} Claudia Choi*1, Claudia Hundertmark², Bernhard Thielen', Beatrice Benkert ${ }^{1}$, Richard Münch ${ }^{1}$, Max Schobert ${ }^{1}$, Dietmar Schomburg ${ }^{3}$, Dieter Jahn ${ }^{1}$ and Frank Klawonn ${ }^{4}$

\author{
Address: ${ }^{1}$ Institut für Mikrobiologie, Technische Universität Braunschweig, Germany, ${ }^{2}$ Helmholtz-Zentrum für Infektionsforschung GmbH, \\ Germany, ${ }^{3}$ Institut für Biochemie, Universität zu Köln, Germany and ${ }^{4}$ Fachbereich Informatik, Fachhochschule Wolfenbüttel, Germany \\ Email: Claudia Choi* - c.choi@tu-bs.de \\ * Corresponding author
}

from BioSysBio 2007: Systems Biology, Bioinformatics and Synthetic Biology

Manchester, UK. II-13 January 2007

Published: 8 May 2007

BMC Systems Biology 2007, I(Suppl I):P20 doi: I0.1 I86/I752-0509-I-SI-P20

This abstract is available from: http://www.biomedcentral.com/I752-0509/I?issue=SI

(c) 2007 Choi et al; licensee BioMed Central Ltd.

Metabolomics emerges as one key aspect of systems biology, since quantifying the dynamic set of metabolites reveals the effect of altered gene expression and protein pattern and thus complements transcriptomics and proteomics. By high-throughput techniques, such as measuring metabolites by gas chromatography-mass spectrometry (GC-MS), enormous data amounts are produced, that need to be analysed. At present, a variety of methods are available for cluster analysis of metabolome data.

Our maximum likelihood approach identifies significantly altered metabolites between Pseudomonas aeruginosa samples grown under different conditions and measured by GC-MS. P. aeruginosa is a versatile soil bacterium and an important opportunistic pathogen causing persistent infection in immunocompromised patients. This statistical approach estimates the inherent noise of the samples and thereby evaluates the significance of altered metabolite composition. Identified key metabolites with significantly altered pattern under different conditions, will be interesting for further investigation of the metabolic network and flux analysis. 